\title{
Zur Einleitung: Lebenswirklichkeiten und politische Konstruktionen in Grenzregionen
}

\author{
Christian Wille
}

Dieser Band erscheint zum 30. Jahrestag der Abschaffung der Personenkontrollen an den EU-Binnengrenzen. Das Schengener Abkommen wurde am 14. Juni 1985 unterzeichnet, und zwar auf dem Schiff MS Princesse-Marie-Astrid. Auf der Mosel fahrend befand es sich zum Zeitpunkt der Signatur im Dreiländereck Deutschland - Frankreich - Luxemburg, genauer gesagt auf der Höhe der luxemburgischen Gemeinde Schengen und nur wenige Meter gegenüber der deutschen Gemeinde Perl. Streng genommen müsste daher auch Perl im Namen des weltweit bekannten Übereinkommens vorkommen. Es ist aber v.a. das Setting des politischen Akts, das für diesen Band interessant ist: Der Schnittpunkt Dreiländereck, der Grenzfluss Mosel und schließlich der >flottierende Boden < des Geschehens sind in ihrer Symbolik kaum zu übertreffen. Der Schauplatz verweist aber zugleich auf das Problem ein Territorium zu finden, das sich nationalstaatlicher Zuordnung entzieht. Dies bestätigt der Umstand, dass das Abkommen schließlich der Gemeinde Schengen und nicht gleichermaßen Perl zugeschlagen wurde. Eine Erklärung dafür könnte die luxemburgische Flagge geben, unter der die Princesse-Marie-Astrid bis heute fährt und die trotz aller Bemühungen dem Geschehen schließlich doch eine nationalstaatliche Rahmung gab.

30 Jahre nach Unterzeichnung des Abkommens, welches mit der Schaffung >offener Grenzen< das nationalstaatliche Ordnungsprinzip relativierte, diskutieren die Autoren ${ }^{1}$ in diesem Band solche oben inszenierten Räume, die sich in nationalstaatlichen Kategorien nur schwer abbilden lassen. Die Betrachtungen bleiben dabei in Grenznähe, d. h. in einer europäischen Grenzregion, entstehen hier doch aufgrund von grenzüberschreitenden Alltagsmobilitäten, Verflechtungen und politischen Beziehungen vermehrt und z. T. sehr anschau-

1 | Die ausschließliche Verwendung der männlichen Form ist in diesem Band geschlechtsunabhängig zu verstehen. Daher wird auf die Nennung der weiblichen Form im Folgenden verzichtet. 
lich zu nationalen Grenzen quer liegende Räume. Mit dieser letzten Formulierung wird deutlich, dass die Auseinandersetzung mit grenzüberschreitenden Räumen einer Mehrebenen-Logik folgen muss, und zwar der territorialen Ordnung nationalstaatlicher Gliederung einerseits und der räumlichen Formation gesellschaftlicher Praxis andererseits. ${ }^{2}$ Dabei fungiert die territoriale Ordnung lediglich als Hintergrundfolie für räumliche Praxisformationen, da sie Räume jenseits nationalstaatlicher Gliederung theoretisch nicht zu denken erlaubt und empirische Wirklichkeiten in Grenzregionen nicht adäquat abzubilden vermag. Dennoch ist die nationalstaatliche Gliederung und die damit verbundene Grenzlage nicht gänzlich auszublenden, wirft sie doch erst die Fragestellung dieses Bands auf und steht mit räumlichen Praxisformationen im Zusammenhang.

Die Beschäftigung mit Räumen jenseits nationalstaatlicher Zuordnungen konzentriert sich in diesem Band allerdings auf räumliche Formationen gesellschaftlicher Praxis. Damit ist eine Verbindungslinie zum Schengener Abkommen gelegt, das neben weiteren juristischen Abkommen und Instrumenten die gesellschaftliche Praxis in Grenzregionen entscheidend beeinflusst. Zu klären bleibt aber, was unter räumlichen Formationen gesellschaftlicher Praxis zu verstehen ist und wie sie erschlossen werden können. Dafür kann an das spätestens seit dem spatial turn etablierte Raumverständnis angeknüpft werden, nach dem Raum als Ergebnis von sozialen (Re-)Produktionsprozessen konzeptualisiert wird (vgl. Wille/Hesse 2014). Demzufolge sind zu nationalen Grenzen quer liegende Räume in Verbindung zu bringen mit sozialen Prozessen, genauer gesagt mit grenzüberschreitender gesellschaftlicher Praxis, die als Kristallisationspunkt für grenzüberschreitende Räume steht. Ihre Untersuchung geht also nicht von vorgängig existenten Räumen aus, sondern sie fokussiert auf die performative Dimension gesellschaftlicher Praxis in Grenzregionen in ihrer räumlichen Strukturiertheit. Aussagen über so erschlossene Räume - die als grenzüberschreitende und dynamische Formationen von Praktiken, Artefakten, Personen, Beziehungen, Wissen und Orten zu denken sind - müssen dann stets Aussagen über die gesellschaftlichen Praktiken ihrer Konstitution sein. Diese bilden in den folgenden Beiträgen den Ausgangspunkt der Überlegungen, wobei die bislang nur unzureichend bearbeitete Frage nach einer fundierten Konzeptualisierung von >grenzüberschreitender gesellschaftlicher Praxis< bzw. >Grenzlandgesellschaft< auch hier unbeantwortet bleiben muss. Jedoch lassen sich zwei basale Untersuchungsperspektiven einnehmen, um empirische Versatzstücke für eine >Soziologie der Grenze < beizusteuern. Dabei handelt es sich um eine bottom-up- und eine top-down-Perspektive, die in diesem

2 | Diese Unterscheidung wird aus didaktischen Gründen vorgenommen. Sie soll nicht den Blick auf territoriale Ordnungen nationalstaatlicher Gliederung als räumliche Formationen gesellschaftlicher Praxis verstellen. 
Band weniger auf das Basis-Überbau-Schema und seine machtkritischen Implikationen abstellen denn vielmehr auf die jeweiligen Akteure und ihre grenzüberschreitenden Raumproduktion. Diese realisieren sich in Prozessen der Zuschreibung, Projektion bzw. intentionalen Konstruktion (top-down) sowie der Aneignung und alltagskulturellen Konstitution (bottom-up). Beide Perspektiven berücksichtigen sowohl institutionelle Akteure als auch Individuen und lassen sich in die Kategorien der grenzüberschreitenden politischen Konstruktion (topdown) und grenzüberschreitenden Lebenswirklichkeiten (bottom-up) übersetzen. Diese stehen allerdings nicht unverbunden nebeneinander, sondern sind miteinander verschränkt und einander bedingend $\mathrm{zu}$ denken. Die getroffene Unterscheidung ist daher analytischer Art und ermöglicht verschiedene Zugänge zu grenzüberschreitender gesellschaftlicher Praxis und damit zur Frage nach Raumproduktionen, die - im Sinne des Schengener Abkommens - nationalstaatliche Ordnungen relativieren oder pointiert formuliert: die nationalstaatliche Rahmungen des Sozialen in Frage stellen.

Die vorgenommenen Grundlegungen werden in diesem Band anhand eines zu nationalen Grenzen quer liegenden Raums exemplifiziert. Dabei handelt es sich um die Großregion SaarLorLux, die erwartungsgemäß von den Autoren nicht als gesetzte Entität vorausgesetzt, sondern als Ergebnis sozialer Prozesse untersucht und kritisch diskutiert wird. Anlass dafür gaben die unten vorzustellenden Konstruktionen, Repräsentationen und lebensweltlichen Erfahrungen, die in ihrer Wirkmächtigkeit, aber auch in ihrer Widersprüchlichkeit, die Frage aufwerfen, wie die Großregion SaarLorLux immer wieder aufs Neue hervorgebracht wird und was sie kennzeichnet.

Gegenstand des Bands ist also die Großregion SaarLorLux als soziale (Re-) Produktion - sowohl auf Ebene der Lebenswirklichkeiten als auch der politischen Konstruktionen. Im institutionellen Diskurs wird sie zumeist projiziert als grenzüberschreitende Region im Herzen Europas mit 11 Millionen Einwohnern, einer Ausdehnung von rd. 65000 km², dem größten Grenzgängeraufkommen in der EU, einer langjährigen Erfahrungen in der grenzüberschreitenden Kooperation und identitätsstiftenden Krisenerfahrungen. Bei der geographischen Bestimmung wird die >hinterlegte< territoriale Ordnung virulent, wenn (im derzeit größten institutionellen Zuschnitt) die deutschen Bundesländer Saarland und Rheinland-Pfalz, die französische Region Lothringen, die belgische Region Wallonien mit der Französischen Gemeinschaft Belgiens und der Deutschsprachigen Gemeinschaft Belgiens sowie der souveräne Nationalstaat Luxemburg zur Großregion SaarLorLux gezählt werden (vgl. Karte 1). Die Kooperationsbeziehungen zwischen diesen Gebietskörperschaften sind ein weiteres und oft als konstitutiv herausgestelltes Merkmal, blicken die politischen Akteure der Großregion SaarLorLux doch auf fast ein halbes Jahrhundert praktizierter grenzüberschreitender Kooperation zurück (vgl. Wille 2012: 119ff.). Dazu zählen die Anfänge der Zusammenarbeit in den frühen 197oer-Jahren, 
die zunächst auf intergouvernementalen Beziehungen zwischen Deutschland, Frankreich und Luxemburg beruhten. Weiter die 1980er- und 9oer-Jahre, die von einer Ausdifferenzierung der Zusammenarbeit auf Ebene der Exekutive und Legislative, der Wirtschafts- und Sozialpartner sowie auf Ebene der Kommunen und Gemeinden geprägt waren bei gleichzeitig verstärkter Institutionalisierung der regionalpolitischen Zusammenarbeit. Ab der Jahrtausendwende entwickelte sich die kommunale Zusammenarbeit weiter und bestehende Kooperationen - insbesondere der Exekutiven - verfestigten sich und wurden weiter professionalisiert (vgl. weiterführend Clément und Evrard/Schulz in diesem Band). Im Vorgriff auf die Beiträge sei hier das höchste politische Gremium der grenzüberschreitenden Zusammenarbeit genannt: der Gipfel der Großregion, der mit dem Erscheinen dieses Bands (2015) sein 20-jähriges Bestehen feiert.

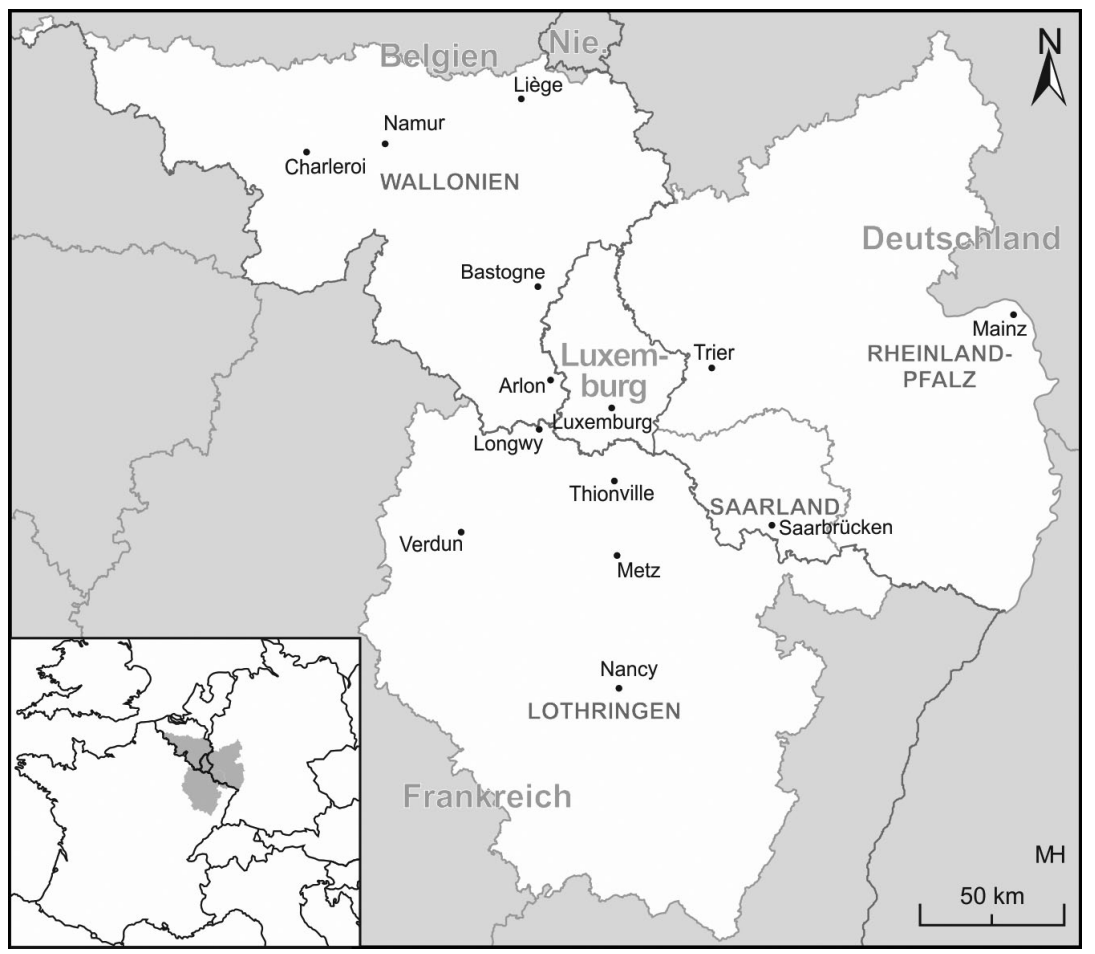

Karte 1: Die institutionelle Großregion SaarLorLux; Entwurf und Kartografie: Malte Helfer

Dem oben gezeichneten Bild der Großregion SaarLorLux sollen nun Lebenswirklichkeiten, d.h. Erfahrungen und Repräsentationen, zur Seite gestellt werden, die in einer Interviewserie bei den Einwohnern des grenzüberschreitenden 
Raums erhoben wurden (vgl. Wille u.a. 2014: 49 ff.). Die 47 Gesprächspartner thematisieren und qualifizieren im Zusammenhang mit dem Begriff > Großregion < verschiedene Aspekte, z. B. den geographischen Zuschnitt der Großregion SaarLorLux: Er wird mit unterschiedlichen Maßstabsebenen (national, regional, kommunal) in Verbindung gebracht, wobei besonders häufig die Länder Luxemburg, Frankreich und Deutschland, die Regionen Province de Luxembourg, Saarland, Rheinland-Pfalz und die Städte Trier, Saarbrücken und Arlon genannt werden (vgl. auch Scholz in diesem Band). Daneben treffen die Befragten diffuse Aussagen über die geographische Ausdehnung, wenn sie »die Städte um Luxemburg herum«, »alles im Umkreis von $100 \mathrm{~km}$ um Luxemburg« oder »ein bisschen Deutschland, ein bisschen Frankreich und ein bisschen Belgien« zur Großregion SaarLorLux zählen.

Ferner berichten die Gesprächspartner (v. a. in Luxemburg), mit dem Begriff >Großregion « »über die Medien« vertraut zu sein. Sie erwähnen bspw. einen Radiosender, der betont, für die Großregion SaarLorLux zu berichten. Außerdem wird auf Gratiszeitungen verwiesen, die in Luxemburg und in den unmittelbar angrenzenden Ortschaften ausliegen (vgl. weiterführend Goulet/Vatter in diesem Band), und in denen »man immer wieder von Großregion liest«. Außerdem wird der Begriff mit dem grenzüberschreitenden Großprojekt Luxemburg und Großregion - Kulturhauptstadt Europas 2007 (vgl. weiterführend Sonntag und Crenn in diesem Band) assoziiert. Während des Kulturhauptstadtjahres ging es aus Sicht des Marketings darum, »Luxemburg im Kontext der Großregion im Sinne eines Zuschreibungsprozesses zu markieren « und der Region eine »(groß-)regionale Identität« (Reddeker 2010: 196 f.) zu verleihen. Angesichts der Interviewergebnisse scheint dies gelungen zu sein.

Der Begriff >Großregion< wird von einigen Befragten auch mit der europäischen und/oder grenzüberschreitenden Zusammenarbeit in Verbindung gebracht, wobei die zahlreichen Gremien der politischen Kooperation (vgl. weiterführend Clément und Evrard/Schulz in diesem Band) nicht namentlich genannt, sondern vielmehr die lebensweltlich erfahrbar werdenden Ergebnisse der Zusammenarbeit angesprochen werden: z. B. der freie Güter-, Dienstleistungs-, Kapital- und Personenverkehr oder grenzüberschreitende Verkehrsprojekte. Ein mit der Personenfreizügigkeit verknüpfter und von den Gesprächspartnern äußerst häufig erwähnter Aspekt ist die Grenzgängerbeschäftigung in Luxemburg (vgl. weiterführend Belkacem/Pigeron-Piroth in diesem Band). Die Befragten in Luxemburg betonen z. B., dass das Großherzogtum »wegen der Grenzgänger « auf die Großregion SaarLorLux angewiesen sei. Umgekehrt wird Luxemburg von den Bewohnern der angrenzenden Regionen als »Arbeitgeber Nummer eins « beschrieben oder als wirtschaftlicher »driver«, der auf die Nachbarregionen abstrahlt. Die Grenzgänger unter den Befragten verbinden mit »Großregion« v.a. das grenzüberschreitende Pendeln, ihre grenzüberschreitenden Kollegenkreise und die Vorteile der Grenzgängerbeschäftigung. 
Daneben evozieren viele Gesprächspartner mit »Großregion« die Möglichkeit, »schnell in einem anderen Land $\mathrm{zu}$ sein«. Sie berichten dabei von grenzüberschreitenden Alltagspraktiken, v. a. vom gezielten Einkauf besonders günstiger Produkte in Luxemburg und mit Blick auf die angrenzenden Regionen vom allgemeinen grenzüberschreitenden Einkaufen bzw. Shoppen (vgl. weiterführend Wille und Scholz in diesem Band) sowie vom Phänomen der grenzüberschreitenden Wohnmigration (vgl. weiterführend Boesen/Schnuer in diesem Band).

Diese schlaglichtartigen Einblicke in Lebenswirklichkeiten zeigen, dass die Einwohner die Großregion SaarLorLux als ein diffuses grenzüberschreitendes Bild variabler räumlicher Verknüpfungen von Städten, Regionen und Ländern repräsentieren. Dieses Bild, das von der institutionellen Großregion SaarLorLux (vgl. Karte 1) deutlich abweicht und sehr vielfältig ist, scheint z. T. von medialen Repräsentationen, politischen Diskursen und von der omnipräsenten Erfahrung der Grenzüberschreitung beeinflusst zu sein.

Schließlich ist auf den Begriff > SaarLorLux < einzugehen, der sowohl von den Befragten in Gesprächen als auch von institutionellen Akteuren in offiziellen Dokumenten z. T. synonym oder komplementär zum Begriff >Großregion < verwendet wird. Ihr diffuser Gebrauch zur Bezeichnung des grenzüberschreitenden Raums war Gegenstand einer Untersuchung, die zeigte, dass >SaarLorLux< bei den Einwohnern - trotz der Dominanz von >Großregion < im politischen Diskurs seit den 1990er-Jahren - populärer ist (vgl. Cavet/Fehlen/Gengler 2006: 24 ff.). Dieser Umstand gab den Akteuren der grenzüberschreitenden Kooperation vermutlich Anlass, im Jahr 2002 eine Bürgerbeteiligung zu organisieren mit dem Ziel, einen neuen Namen für die »Großregion« zu finden, der »unverwechselbar ist und dem territorialen Bild unserer Region und ihrem Selbstverständnis Rechnung trägt« (Staatskanzlei des Saarlandes 2002). Im Rahmen dieser Initiative, die als (Versuch einer) Raumkonstruktion qualifiziert werden kann, wurden 3000 Namen eingereicht, die an eine >gemeinsame Geschichte< des grenzüberschreitenden Raums oder an geografische Besonderheiten anknüpften: z. B. Carolinga, Lotharingia, Centregio, Centropa oder Rhesamemo. Letztes Akronym für RheinSaarMeuseMosel erzielte zwischen den politischen Akteuren einen Minimalkonsens, es konnte sich dennoch nicht durchsetzen (vgl. Chambre des Députés du Grand-duché de Luxembourg 2003). Schließlich wurde keiner der Vorschläge ausgewählt und die politisch Verantwortlichen vereinbarten, »dass die in den einzelnen Teilräumen üblichen Bezeichnungen weiter verwendet werden können« (Gipfel der Großregion 2003: 18).

So ist bis heute eine Gleichzeitigkeit der Begriffe >SaarLorLux < und >Großregion< verbreitet, die die Identifizierung mit und die Identifikation von dem grenzüberschreitenden Raum keineswegs befördert, aber mit wirtschaftlichen und politischen Entwicklungen erklärbar ist (vgl. Wille 2009). So wurde das Kürzel >SaarLorLux< von Hubertus Rolshoven geprägt, der damit im Jahr 1969 
erstmals ein grenzüberschreitendes Gebiet mit ähnlicher Industriestruktur bezeichnete (vgl. weiterführend Helfer in diesem Band). Fortan verwies >SaarLorLux auf den im Saarland, Lothringen und Luxemburg sowohl sozioökonomischen als auch soziokulturell bedeutsamen Steinkohlenbergbau und die Stahlindustrie. Mit dem Untergang der Montanindustrie aber (vgl. weiterführend Dörrenbächer in diesem Band) schlossen sich die politischen Akteure der damaligen SaarLorLux-Region zusammen, um gemeinsam Wege aus der schwierigen Lage zu finden. Der Kreis der grenzüberschreitenden Kooperationspartner erweiterte sich jedoch in den Folgejahren um Rheinland-Pfalz und später um Wallonien mit seinen Sprachgemeinschaften (vgl. weiterführend Clément in diesem Band). Die Semantik des Kürzels >SaarLorLux < geriet somit an seine Grenzen, weshalb die politischen Akteure in den 199oer-Jahren den umfassenderen Begriff >Großregion< einführten und stark machten. Trotz dieser top-down-Maßnahme erweist sich >SaarLorLux< - als Topos der Wirtschafts- und Sozialgeschichte des grenzüberschreitenden Raums - bis heute als resistent in den Lebenswirklichkeiten der Einwohner, was auch mit der viel diskutierten Semantik von >Großregion< verknüpft zu sein scheint (vgl. weiterführend Schönwald und Scholz in diesem Band). Die Autoren in diesem Band operieren mit der Bezeichnung >Großregion SaarLorLux <, die die Betrachtungen nicht verengt auf Saarland, Lothringen und Luxemburg, sondern die die oben beschriebenen politischen Konstruktionen und Lebenswirklichkeiten zusammendenkt und benennt. Im Gegensatz dazu zeigt die Verwendung des Begriffs >SaarLorLuxRaum < in diesem Band eine Fokussierung der Betrachtungen an, und zwar auf die mit dem Kürzel aufgerufenen Territorien.

Die 16 Beiträge des Bands, so ist schließlich festzuhalten, thematisieren die Großregion SaarLorLux als soziale (Re-)Produktionen, wofür einige der oben angerissenen politischen Konstruktionen und Lebenswirklichkeiten - sowie darüber hinaus - als mögliche Zugänge zu grenzüberschreitenden Praxisformationen genutzt werden. Über dieses Vorgehen gelangen die Autoren zu Antworten auf die generelle Frage, wie zu nationalen Grenzen quer liegende Räume entstehen und beschaffen sind, bzw. auf die hier spezifische Frage, wie die Großregion SaarLorLux immer wieder aufs Neue konstituiert wird und was sie ausmacht. Dafür untersuchen die Autoren fünf thematische Bereiche grenzüberschreitender gesellschaftlicher Praxis, die jeweils verstanden wird als Kristallisationspunkt eines grenzüberschreitenden Wirtschafts- und Beschäftigungsraums, politischen Handlungsraums, Lebens- und Identitätsraums, medialen Kommunikationsraums und Kulturraums.

In dem Band kommen 19 Autoren aus den Ländern Deutschland, Frankreich und Luxemburg zu Wort, von denen viele auch im Rahmen des 2014 gegründeten UniGR-Center for Border Studies zusammenarbeiten. Allen Autoren gilt mein Dank für die inspirierende Zusammenarbeit und für die Bereitschaft, sich aus unterschiedlichen disziplinären Blickwinkeln auf die Fragestellung des 
Bands einzulassen. Darüber hinaus möchte ich mich bei Malte Helfer und Edwina von der Wense bedanken, die bei der Anfertigung des Kartenmaterials und der Vorbereitung des Manuskripts eine große und kollegiale Unterstützung waren. Für die großzügige Finanzierung und ideelle Unterstützung des Buchprojekts danke ich Heinz Sieburg und Sandra Baumann (Universität Luxemburg) sowie Luciënne Blessing und Kristina Hondrila (Universität der Großregion).

\section{Literatur}

Cavet, Marine/Fehlen, Fernand/Gengler, Claude (2006): Leben in der Großregion. Studie der grenzüberschreitenden Gewohnheiten in den inneren Grenzräumen der Großregion SaarLorLux/Rheinland-Pfalz/Wallonien. Luxemburg: Saint-Paul.

Chambre des Députés du Grand-duché de Luxembourg: Réponse à la question parlementaire, Nr. 2053 vom 24. Februar 2003, Luxemburg.

Gipfel der Großregion (2003): Gemeinsame Erklärung. Saarbrücken.

Reddeker, Sebastian (2011): Werbung und Identität im multikulturellen Raum. Der Werbediskurs in Luxemburg. Ein kommunikationswissenschaftlicher Beitrag. Bielefeld: transcript Verlag.

Staatskanzlei des Saarlandes (2002): Ein Name für die Region. Pressemitteilung vom 22. Mai.

Wille, Christian (2009): Eine namenlose Region. In: Forum für Politik, Gesellschaft und Kultur in Luxemburg 288, S. 30 f.

Wille, Christian (2012): Grenzgänger und Räume der Grenze. Raumkonstruktionen in der Großregion SaarLorLux. Frankfurt a. M.: Peter Lang.

Wille, Christian/Hesse, Markus (2014): Räume: Zugänge und Untersuchungsperspektiven. In: Christian Wille/Rachel Reckinger/Sonja Kmec/Markus Hesse (Hg.): Räume und Identitäten in Grenzregionen. Politiken - Medien - Subjekte. Bielefeld: transcript Verlag, S. 24-35.

Wille, Christian/Reckinger, Rachel/Kmec, Sonja/Hesse, Markus (Hg.; 2014): Räume und Identitäten in Grenzregionen. Politiken - Medien - Subjekte. Bielefeld: transcript Verlag. 


\section{Christian Wille (Hg.)}

\section{Lebenswirklichkeiten und}

\section{politische Konstruktionen}

in Grenzregionen

Das Beispiel der Großregion SaarLorLux Wirtschaft - Politik - Alltag - Kultur

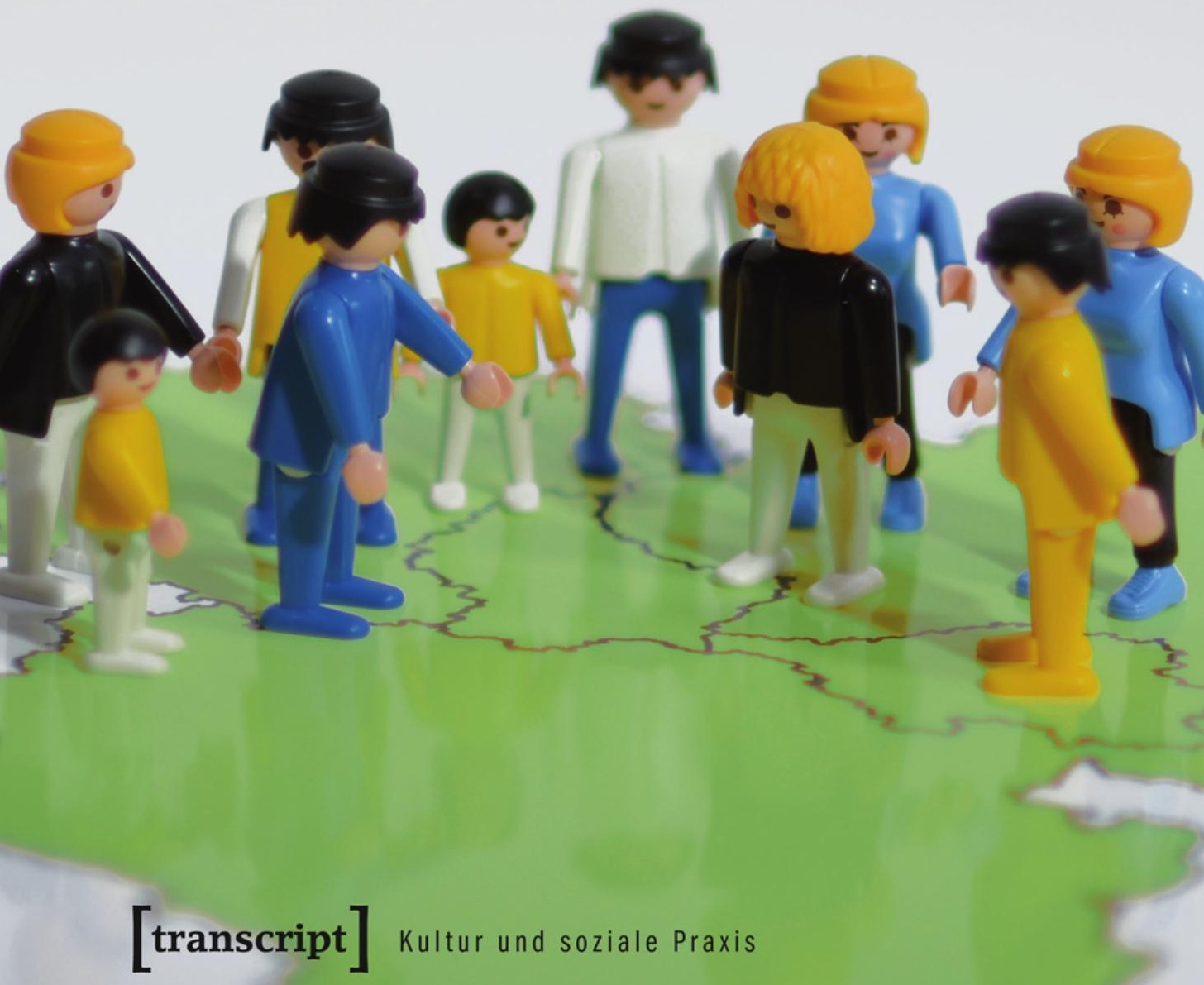




\section{Aus:}

Christian Wille (Hg.)

\section{Lebenswirklichkeiten und politische Konstruktionen in Grenzregionen}

Das Beispiel der Großregion SaarLorLux:

Wirtschaft - Politik - Alltag - Kultur

August 20I5, 348 Seiten, kart., zahlr. z.T. farb. Abb., 34,99 €, ISBN 978-3-8376-2927-9

Wie entstehen grenzüberschreitende Regionen und was charakterisiert sie?

Am Beispiel der Großregion SaarLorLux untersuchen die aus Deutschland, Frankreich und Luxemburg stammenden Beiträger_innen dieses Bandes die gesellschaftliche Praxis an EU-Binnengrenzen. Sie diskutieren die Praktiken von institutionellen Akteuren und von Grenzraumbewohnern in den Bereichen Wirtschaft, Arbeitsmarkt, politische Kooperation sowie Alltag, Medien und Kultur.

Die sozial- und kulturwissenschaftlichen Beiträge situieren die Großregion SaarLorLux zwischen grenzüberschreitenden Lebenswirklichkeiten und politischen Konstruktionen.

Christian Wille (Dr.) ist Sozial- und Kulturwissenschaftler an der Universität Luxemburg.

Weitere Informationen und Bestellung unter: www.transcript-verlag.de/978-3-8376-2927-9 


\section{Inhalt}

Zur Einleitung: Lebenswirklichkeiten und politische Konstruktionen in Grenzregionen

Christian Wille | IX

\section{WIRTSChAFt UND GRENZÜBERSChreItender ARbeItSmarkt}

Die Industrialisierung der Großregion SaarLorLux

Malte Helfer | 3

Ein grenzüberschreitender Wirtschaftsraum?

Gemeinsame und individuelle Entwicklungen

seit der Krise des Montansektors

H. Peter Dörrenbächer 21

Un marché de l'emploi intégré ?

L'emploi frontalier et ses dimensions socio-économiques

Rachid Belkacem/Isabelle Pigeron-Piroth | 39

\section{GoVERnANCE UND GRENZÜBERSCHREITENDE ZUSAMMENARBEIT}

La représentation politique dans la Grande Région SaarLorLux :

institutions, compétences et coopérations

Franz Clément | 61

Vers une région métropolitaine polycentrique?

Enjeux d'un aménagement du territoire transfrontalier

Estelle Evrard/Christian Schulz | 83

Alle an einem Strang?

Zur Rolle von Identitäten und Stereotypen in der grenzüberschreitenden Kooperation

Antje Schönwald | 107 


\section{Grenzüberschreitende Alltagspraktiken Und Identitäten}

Grenzüberschreitende Alltagspraktiken in der Großregion SaarLorLux: eine Bestandsaufnahme

Christian Wille | 133

Die Großregion SaarLorLux zwischen Vision und Wirklichkeit: grenzüberschreitende Alltagspraktiken und Raumbilder von Jugendlichen

Gundula Scholz | 157

Wohnen jenseits der Grenze.

Regionale Integration und ihre lokalen Verwirklichungen

Elisabeth Boesen/Gregor Schnuer | 179

\section{MEDIENPRAKTIKEN UND GRENZÜBERSCHREITENDE MEDIENÖFFENTLICHKEITEN}

Grenzüberschreitende Medienöffentlichkeiten?

Zur Berichterstattung im SaarLorLux-Raum am Beispiel von , Migration،

Elena Kreutzer | 205

L'espace médiatique transfrontalier : médias, flux d'informations et pratiques journalistiques Vincent Goulet/Christoph Vatter | 231

\section{KUNST UND GRENZÜBERSCHREITENDE KULTURPOLITIK}

"Für uns ist die grenzüberschreitende Zusammenarbeit eine Selbstverständlichkeit ..." - die Großregion SaarLorLux aus Sicht von Kulturakteuren

Monika Sonntag $\mid 253$

Stratégies et dispositifs culturels dans la construction d'un espace culturel. Les politiques muséales de Luxembourg et Grande Région - Capitale européenne de la culture 2007 Gaëlle Crenn | 273 
Europäische Kulturgemeinschaft:

Kristallglasmacher in der Großregion - Grande Région:

une communauté culturelle du savoir-faire

Eva Mendgen | 295

\section{SCHLUSSBETRACHTUNG}

Die Großregion SaarLorLux: Lebenswirklichkeiten und politische Konstruktionen

Birte Nienaber | 311

Zusammenfassungen der Beiträge | 315

Autorinnen und Autoren | 327 